\title{
Maternal HIV infection and other factors associated with growth outcomes of HIV-uninfected infants in Entebbe, Uganda
}

\author{
Lawrence Muhangi ${ }^{1}$ *, Swaib A Lule ${ }^{1}$, Harriet Mpairwe ${ }^{1}$, Juliet Ndibazza ${ }^{1}$, \\ Moses Kizza ${ }^{1}$, Margaret Nampijia ${ }^{1}$, Esther Nakazibwe ${ }^{1}$, Macklyn Kihembo', \\ Alison M Elliott ${ }^{1,2}$ and Emily L Webb ${ }^{2}$ \\ 'Medical Research Council/Uganda Virus Research Institute (MRC/UVRI) Uganda Research Unit on AIDS, \\ PO Box 49, Plot 51-59 Nakiwogo Road, Entebbe, Uganda: ${ }^{2}$ London School of Hygiene and Tropical Medicine, \\ London, UK
}

Submitted 20 October 2011: Final revision received 12 November 2012: Accepted 23 January 2013: First published online 18 March 2013

\begin{abstract}
Objective: To assess the associations between maternal HIV infection and growth outcomes of HIV-exposed but uninfected infants and to identify other predictors for poor growth among this population.

Design: Within a trial of de-worming during pregnancy, the cohort of offspring was followed from birth. HIV status of the mothers and their children was investigated and growth data for children were obtained at age 1 year. Lengthfor-age, weight-for-age and weight-for-length $Z$-scores were calculated for each child; $Z$-scores $<-2$ were defined as stunting, underweight and wasting, respectively.

Setting: The study was conducted in Entebbe municipality and Katabi subcounty, Uganda.

Subjects: The sample consisted of 1502 children aged 1 year: HIV-unexposed ( $n$ 1380) and HIV-exposed not infected ( $n$ 122).

Results: Prevalence of stunting, underweight and wasting was $14 \cdot 2 \%, 8 \cdot 0 \%$ and $3.9 \%$, respectively. There was evidence for an association between maternal HIV infection and odds of being underweight (adjusted OR $=2 \cdot 32 ; 95 \%$ CI 1.32, 4.09; $P=0.006$ ) but no evidence for an association with stunting or with wasting. Young maternal age, low maternal education, low birth weight, early weaning and experiencing a higher number of episodes of malaria during infancy were independent predictors for stunting and underweight. A higher number of living children in the family was associated with wasting.

Conclusions: Maternal HIV infection was associated with being underweight in HIV-exposed uninfected infants. The success of programmes for prevention of mother-to-child HIV transmission means that an increasing number of infants will be born to HIV-infected women without acquiring HIV. Therefore, viable nutritional interventions need to be identified for this population.
\end{abstract}

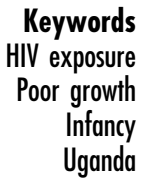

Growth failure in children is a global health problem in developing countries. Data on predictors of growth failure in infants born to women infected with HIV are limited in this setting ${ }^{(1)}$. In vertically HIV-infected children, poor growth may be an early marker of infection or progression to disease ${ }^{(2)}$. HIV-infected children normally grow considerably more slowly than their HIV-exposed, uninfected counterparts, and the gap between the two groups tends to widen with age. Children born to HIVpositive women are particularly susceptible to malnutrition. Growth failure is frequently found in this population, especially in areas where a high prevalence of HIV/AIDS coexists with high rates of food insecurity ${ }^{(2,3)}$.
HIV exposure in utero without subsequent infection may affect growth in infancy and early childhood ${ }^{(2)}$. Newborns whose mothers are infected with HIV may have higher rates of fetal malnutrition than newborns of HIV-seronegative mothers ${ }^{(4)}$, and this disadvantage is likely to extend beyond infancy. A study in Zambia found that HIV-exposed, uninfected infants had poorer growth than their HIV-unexposed counterparts ${ }^{(5)}$. According to a WHO working group, uninfected infants born to HIVpositive mothers grow in an unfavourable intra-uterine environment where, depending upon the setting, they may suffer from greater exposure to maternal infection and maternal malnutrition, or to drug addiction, smoking 
and poor social conditions ${ }^{(6)}$. Furthermore, maternal HIV severity is associated with higher morbidity and mortality, and lower weight at age 4 months, among HIV-exposed but uninfected children ${ }^{(7)}$. More studies have been recommended to elucidate the relationship between maternal immune status markers including viral load and CD4 cell count and child growth ${ }^{(8)}$.

Many other determinants of nutritional status of children have been reported. Parental education, particularly mother's education, is reported to have a strong influence on the nutritional status of the child ${ }^{(9-11)}$. Household socioeconomic status and access to clean water may also have a significant impact on children's nutritional status ${ }^{(11,12)}$. Some studies have found that rural children are more likely to be stunted, wasted or underweight than those living in urban settings ${ }^{(9,13)}$. Maternal health is also important, for example maternal depression may interfere with the quality of care given to the infant ${ }^{(14)}$. In addition birth order of the child has been associated with poor growth; the higher the birth order, the higher the risk of malnutrition ${ }^{(15)}$.

The main aims of the present analysis were to: (i) assess the associations between maternal HIV infection and growth outcomes of HIV-exposed but uninfected infants; and (ii) identify other potential determinants for poor growth among infants in Entebbe municipality and Katabi sub-county of Uganda.

\section{Materials and methods}

\section{Study area and participants}

The study was conducted in Entebbe municipality and Katabi sub-county as previously described ${ }^{(16)}$. It was a randomized, double-blind, placebo-controlled trial of anthelminthics in pregnancy, using albendazole $v$. placebo and praziquantel $v$. placebo in a $2 \times 2$ factorial design. The study was originally designed to examine effects of anthelminthic treatment during pregnancy on infant responses to immunization and infectious disease incidence. Results of the trial have been reported elsewhere and no effect of the trial intervention on growth parameters was observed ${ }^{(17,18)}$. The study results reported herein represent a retrospective observational analysis of differences in growth at 1 year of age between HIV-exposed but uninfected infants and infants born to women who were HIV-negative. Socio-economic, maternal and infant characteristics were also evaluated for associations with growth outcomes at 1 year of age.

Women were recruited at the antenatal clinic at Entebbe Hospital between April 2003 and November 2005. They were eligible if they were well, resident in the study area, planning to deliver their baby at the hospital, willing to participate and willing to know their HIV status; and excluded if they had $\mathrm{Hb}<8 \mathrm{~g} / \mathrm{dl}$, clinically apparent severe liver disease, diarrhoea with blood in the stool, abnormal pregnancy, history of adverse reaction to anthelminthic drugs or had participated in the study during an earlier pregnancy.
Following delivery, children were followed up according to the study protocol. Morbidity data were collected prospectively at the research clinic. Malaria was diagnosed as fever $\left(\geq 37 \cdot 5^{\circ} \mathrm{C}\right)$ with Plasmodium falciparum parasitaemia. Data on asymptomatic parasitaemia were collected annually. Study nurses were trained on how to take and record the anthropometric measurements. Weight measurements for 1-year-olds were taken using CMS Weighing Equipment, model MP25 (Chasmors Ltd, London, UK). The children were lightly dressed wearing undergarments only, and wore a measuring trouser which was then suspended on a weighing scale. Measurement of recumbent length at age 1 year was done using an adjustable child-length measuring board (Seca; Vogel \& Halke, Hamburg, Germany).

In 2002, prior to the start of the study, the research team in collaboration with Entebbe Hospital established a programme for prevention of mother-to-child HIV transmission at the hospital. In accordance with guidelines current at the time ${ }^{(19)}$, women identified as HIV-positive were offered a single dose of nevirapine for themselves and their infants, to be taken during labour and after delivery, respectively. HIV-positive women were provided with cotrimoxazole daily for prophylaxis of opportunistic infections; HIV-exposed infants were provided with cotrimoxazole syrup from age 6 weeks until their HIV status was assessed at age 18 months; at this time, cotrimoxazole was discontinued for HIV-negative children. HIV-positive infants were referred for care at nearby specialist centres. Highly active antiretroviral therapy (HAART) for treatment was not widely available in Uganda at the time when women were recruited to the study; however, five HIV-positive women were recorded as taking HAART during pregnancy.

\section{HIV diagnosis}

Mothers' HIV status was assessed by a rapid test algorithm before delivery as previously described ${ }^{(20)}$. Vertical HIV transmission was diagnosed by RNA and DNA PCR at age 6 weeks as previously described ${ }^{(18)}$ and by rapid test at age 18 months. Infants were regarded as being HIVpositive if the 6-week sample had a positive DNA PCR for any of the viral regions examined and a viral load of 1000 copies per millilitre or more, or if the rapid test at 18 months of age was positive.

Blood samples were obtained from HIV-infected pregnant women. CD4 cell counts were ordered as part of the baseline measurement and done using a BD FACScount $^{\mathrm{TM}}$ flow cytometer (Becton Dickinson, San Jose, CA, USA). Maternal immunological status was dichotomized based upon results of $\mathrm{CD} 4$ testing performed at enrolment, with women having a CD 4 count of $\leq 350$ cells $/ \mathrm{mm}^{3}$ being categorized as having poor immunological status.

\section{Outcome measures}

The outcome variables for the present study were the three continuous anthropometric measures, length-for-age $Z$-score (LAZ), weight-for-age $Z$-score (WAZ) and weight-for-length 
$Z$-score (WLZ), derived by importing growth parameters into WHO Anthro software version 3 (April 2009). The continuous value of each of the three anthropometric measures was also used to derive three binary variables reflecting the presence or absence of a $Z$-score $<-2$, i.e. stunting (LAZ $<-2$ ), underweight (WAZ $<-2$ ) and wasting (WLZ $<-2$ ), which were then also used as dependent variables for the study objectives.

\section{Statistical methods}

Data management was done using the Microsoft ${ }^{\circledR}$ Access database designed for the anthelminthic trial. Statistical analyses were performed using the STATA statistical software package version 10 . The second ${ }^{(17)}$ or third infant ${ }^{(1)}$ in a twin or triplet pregnancy was excluded from the analysis but five women on HAART were retained.

In order to evaluate the associations between HIV exposure, other factors and growth outcomes, we used as response variables the continuous $Z$-scores and the binary variables for stunting, underweight and wasting defined above.

Univariable analysis was first performed. For the continuous outcomes linear regression was used to investigate whether HIV exposure and other risk factors were associated with the mean $Z$-score; mean differences in $Z$-score and $95 \%$ confidence intervals were used to quantify the association between each risk factor and growth. For the binary outcomes, logistic regression was used to investigate whether HIV exposure and other risk factors were associated with the prevalence of stunting, underweight and wasting; odds ratios were used to quantify the association between each risk factor and poor growth. Multivariable analyses were then conducted investigating factors considered likely to be potential confounders, including maternal age, maternal education, maternal income, household socio-economic status (a score based on building materials, number of rooms and items owned) and episodes of malaria during infancy; variables assumed to be potential confounders and variables that presented a $P$ value of $<0.05$ in the univariable analysis were included in multivariable regression models. Significance levels in the final model were determined using likelihood ratio tests. A similar approach was used to investigate other risk factors for stunting, underweight and wasting in this population. The following factors were considered: sex of the child, low birth weight, low maternal CD 4 cell count, number of living children in the family and early weaning (defined as introducing cow's milk at or before the age of 6 weeks).

\section{Results}

There were 2507 mothers enrolled and 2345 live births, with 2092 children under follow-up throughout the first year of life ${ }^{(18)} ; 1701$ children were seen at age 1 year, of whom 1554 had data on HIV exposure status and growth parameters. Retaining only one infant from twin or triplet pregnancies reduced this number to 1536. Mothers of children who did not have growth data available were, on average, younger than mothers whose children did have growth data $(P<0 \cdot 001)$, more likely to be single mothers $(P=0 \cdot 001)$, more likely to be HIV-positive $(P<0 \cdot 001)$ and more likely to have malaria at enrolment $(P<0 \cdot 001)$ There were no other differences between mothers whose children provided growth data at 1 year and mothers whose children did not contribute to growth data (data not shown).

Of the 1536 children, $1380(89 \cdot 8 \%)$ were not exposed to HIV, $122(8.0 \%)$ were exposed but not infected, nineteen $(1.2 \%)$ were exposed and infected, fifteen $(1 \cdot 0 \%)$ were exposed but their HIV infection status was not available. Of the nineteen exposed and infected, twelve infants tested positive for HIV at 6 weeks and at 18 months, five infants tested negative at 6 weeks but were HIV-positive at 18 months, two infants did not have a 6-week test result but tested positive for HIV at 18 months. The infected children (nineteen) and those whose status was not available (fifteen) were excluded, leaving a total of 1502 children for the present analysis.

Characteristics of the study participants, stratified by HIV exposure status, are shown in Table 1. HIV-positive mothers were more likely to be older, to have primary or no formal education, to be widowed/divorced/separated and to have had five or more pregnancies than HIVnegative mothers in this population. The CD4 count at enrolment ranged from 19 to $1473 \mathrm{cells} / \mathrm{mm}^{3}$, with a median of 538 (interquartile range $345-710$ ) cells $/ \mathrm{mm}^{3}$. HIVexposed uninfected children had a significantly lower mean birth weight than unexposed children $(P=0 \cdot 028)$.

\section{Associations between maternal HIV and growth outcomes}

The overall mean LAZ was $-0 \cdot 82$, WAZ was -0.31 and WLZ was $0 \cdot 12$. Prevalence of stunting, underweight and wasting was $14 \cdot 2 \%, 8 \cdot 0 \%$ and $3 \cdot 9 \%$, respectively. At age 1 year, HIV-exposed uninfected babies had a lower mean LAZ (adjusted mean difference $-0 \cdot 16 ; 95 \%$ CI $-0 \cdot 39,0 \cdot 06$; $P=0 \cdot 16$; Table 2) and a somewhat lower mean WAZ than HIV-unexposed babies (adjusted mean difference $-0 \cdot 13$; $95 \%$ CI $-0 \cdot 35,0 \cdot 09 ; P=0 \cdot 23$; Table 3 ). There was also little difference between mean WLZ in the two groups (adjusted mean difference $-0 \cdot 04 ; 95 \%$ CI $-0 \cdot 27,0 \cdot 19 ; P=0 \cdot 74$; Table 4). Analysis of the binary variables showed that HIV exposure was significantly associated with underweight ( $P=0 \cdot 006$; Table 3 ), but not with stunting or wasting. In other words, HIV-exposed but uninfected children tended to be shorter than HIV-unexposed infants, but their weight was proportionate to their length.

\section{Associations between other factors and growth outcomes}

Young maternal age, low maternal education, low birth weight, male sex and repeated episodes of malaria in 
Table 1 Maternal and child characteristics for HIV-unexposed and HIV-exposed but uninfected infants, Entebbe municipality and Katabi sub-county, Uganda, 2003-2005

\begin{tabular}{|c|c|c|c|c|c|}
\hline \multirow[b]{2}{*}{ Characteristic } & \multicolumn{2}{|c|}{ HIV-unexposed children ( $n$ 1380) } & \multicolumn{2}{|c|}{ HIV-exposed, uninfected ( $n$ 122) } & \multirow[b]{2}{*}{$\begin{array}{l}P \text { value } \\
\left(\chi^{2} \text { test }\right)\end{array}$} \\
\hline & $\begin{array}{l}\text { Mean, } n \\
\text { or median }\end{array}$ & $\begin{array}{l}\text { SD, } \% \\
\text { or IQR }\end{array}$ & $\begin{array}{l}\text { Mean, } n \\
\text { or median }\end{array}$ & $\begin{array}{l}\text { SD, } \% \\
\text { or IQR }\end{array}$ & \\
\hline \multicolumn{6}{|l|}{ Maternal characteristics } \\
\hline Mother's age (years)* & $23 \cdot 78$ & $5 \cdot 36$ & $26 \cdot 35$ & $5 \cdot 43$ & $<0.001$ \\
\hline \multicolumn{6}{|l|}{ Maternal age group (years) $\dagger$} \\
\hline $14-19$ & 327 & 24 & 12 & 10 & \multirow{5}{*}{$<0.001$} \\
\hline $20-24$ & 526 & 38 & 36 & 30 & \\
\hline $25-29$ & 314 & 23 & 42 & 34 & \\
\hline $30-34$ & 148 & 11 & 21 & 17 & \\
\hline$\geq 35$ & 65 & 5 & 11 & 9 & \\
\hline \multicolumn{6}{|l|}{ Mother's educationt } \\
\hline Primary or none & 715 & 52 & 83 & 68 & \multirow[t]{2}{*}{0.001} \\
\hline Secondary or tertiary & 661 & 48 & 39 & 32 & \\
\hline \multicolumn{6}{|l|}{ Marital status $(1 \mathrm{MV}) t$} \\
\hline Single & 161 & 12 & 11 & 9 & \multirow[t]{3}{*}{$<0.001$} \\
\hline Married & 1190 & 86 & 100 & 82 & \\
\hline Widowed/divorced/separated & 28 & 2 & 11 & 9 & \\
\hline \multicolumn{6}{|l|}{ Number of pregnanciest } \\
\hline 1 & 362 & 26 & 18 & 15 & \multirow[t]{5}{*}{0.003} \\
\hline $2-4$ & 802 & 58 & 74 & 61 & \\
\hline$\geq 5$ & 216 & 16 & 30 & 24 & \\
\hline CD4 count at delivery (cells $\left./ \mathrm{mm}^{3}\right) \ddagger$ & - & - & 538 & $345-710$ & \\
\hline \multicolumn{5}{|l|}{ Child characteristics } & \\
\hline \multicolumn{6}{|l|}{ Child's sext } \\
\hline Male & 709 & 51 & 57 & 47 & \multirow[t]{2}{*}{0.324} \\
\hline Female & 671 & 49 & 65 & 53 & \\
\hline Birth weight $(\mathrm{kq})^{*}$ & 3.41 & 0.92 & $3 \cdot 22$ & 0.72 & 0.028 \\
\hline \multicolumn{6}{|l|}{ Birth weight category $(12 \mathrm{MV}) \dagger$} \\
\hline Normal, $\geq 2.5 \mathrm{~kg}$ & 1290 & 94 & 109 & 90 & \multirow[t]{2}{*}{0.068} \\
\hline Low, $<2.5 \mathrm{~kg}$ & 79 & 6 & 12 & 10 & \\
\hline
\end{tabular}

MV, missing values.

*Values are presented as mean and standard deviation.

+Values are presented as number and percentage.

$\ddagger$ Values are presented as median and interquartile range (IQR).

infancy were associated with an increased odds of stunting (Table 2), while being a male child, low birth weight, early weaning and having malaria during infancy were associated with being underweight (Table 3). Higher number of living children in the family, low birth weight and low maternal income were associated with wasting (Table 4). Maternal helminth infection was not associated with poor growth in this population (data not shown); as previously reported, anthelminthic treatment showed no effect on growth during infancy ${ }^{(18)}$.

Associations between growth outcomes and maternal CD4 cell count were explored among infants of HIVpositive mothers by categorizing enrolment CD 4 count into two categories: $\leq 350$ cells $/ \mathrm{mm}^{3}$ and $>350$ cells $/ \mathrm{mm}^{3}$ (Tables 2, 3 and 4). Low maternal CD4 cell count increased the odds of both stunting and underweight but the association was not significant (Tables 2 and 3). The average breast-feeding duration for HIV-unexposed infants (mean 16.73 (SD 5.48) months) was significantly longer than that for HIV-exposed uninfected infants (mean 7.80 (sD 6.07) months; $P<0 \cdot 001$ ). Results from subgroup analysis showed that breast-feeding duration was not associated with growth outcomes.

\section{Discussion}

We have observed that maternal HIV infection is strongly associated with increased odds of being underweight, but not with stunting or wasting, among HIV-exposed uninfected infants in this population. The underlying mechanism is not fully understood and may be multifactorial. The association with underweight but not stunting or wasting indicates chronic nutritional deficiency, as opposed to a sudden effect of acute illness or deprivation. Our study finding that HIV exposure is associated with underweight among HIV-exposed uninfected children corroborates the findings of other studies from Sub-Saharan Africa ${ }^{(5)}$.

Growth faltering among HIV-exposed but uninfected infants could be caused indirectly by HIV-related symptoms in the mother: HIV may incapacitate the mother and this might translate into less provision of care to her child. HIV-positive women with low CD4 cell counts may be at greater risk of postnatal depression ${ }^{(21,22)}$, and this could also influence the care that they are able to provide for their children at a critical stage in their development. Our analysis on measures of maternal immune status showed 
Table 2 Association of HIV exposure and other factors with stunting* in 1-year-olds, Entebbe municipality and Katabi sub-county, Uganda, 2003-2005

\begin{tabular}{|c|c|c|c|c|c|c|c|c|c|c|c|c|c|}
\hline \multirow[b]{2}{*}{ Risk factor } & \multirow[b]{2}{*}{$n$} & \multicolumn{2}{|c|}{ LAZ } & \multicolumn{2}{|c|}{ Difference in LAZ } & \multirow{2}{*}{$\begin{array}{c}P \text { value } \\
\text { (trend) }\end{array}$} & \multicolumn{2}{|c|}{ Stunted } & \multicolumn{4}{|c|}{ Odds for stunting } & \multirow{2}{*}{$\begin{array}{l}P \text { value } \\
\text { (trend) }\end{array}$} \\
\hline & & Mean & SD & Adjusted differencet & $95 \% \mathrm{Cl}$ & & $n / N$ & $\%$ & Unadjusted OR & $95 \% \mathrm{Cl}$ & Adjusted ORt & $95 \% \mathrm{Cl}$ & \\
\hline \multicolumn{14}{|l|}{ HIV exposure } \\
\hline HIV-unexposed & 1362 & $-0 \cdot 81$ & $1 \cdot 19$ & - & - & & $187 / 1356$ & $13 \cdot 8$ & 1.00 & - & 1.00 & - & \\
\hline HIV-exposed & 121 & -0.94 & $1 \cdot 30$ & $-0 \cdot 16$ & $-0.39,0.06$ & $0 \cdot 157$ & $23 / 121$ & $19 \cdot 0$ & $1 \cdot 47$ & $0.91,2 \cdot 37$ & 1.55 & $0 \cdot 92,2 \cdot 61$ & $0 \cdot 107$ \\
\hline \multicolumn{14}{|l|}{ Sex of child } \\
\hline Male & 754 & -0.99 & $1 \cdot 24$ & - & - & & $147 / 753$ & $19 \cdot 5$ & 1.00 & - & 1.00 & - & \\
\hline Female & 729 & -0.65 & $1 \cdot 13$ & 0.33 & $0.22,0.46$ & $<0.001$ & $63 / 724$ & $8 \cdot 7$ & 0.39 & $0.29,0.54$ & 0.37 & $0.26,0.51$ & $<0.001$ \\
\hline \multicolumn{14}{|c|}{ Mother's age group (years) } \\
\hline $14-19$ & 335 & $-1 \cdot 08$ & $1 \cdot 23$ & - & - & $<0.001$ & $66 / 331$ & $19 \cdot 9$ & 1.00 & - & 1.00 & - & 0.002 \\
\hline $20-24$ & 555 & -0.84 & $1 \cdot 18$ & $0 \cdot 26$ & $-0.10,0.43$ & $<0.001$ & $84 / 554$ & $14 \cdot 8$ & $0 \cdot 70$ & $0.49,0.99$ & 0.69 & $0 \cdot 48,1 \cdot 01$ & 0.001 \\
\hline $25-29$ & 350 & -0.65 & 1.05 & $0 \cdot 41$ & $0.22,0.59$ & & $30 / 350$ & $8 \cdot 6$ & 0.38 & $0.24,0.60$ & 0.38 & $0.23,0.63$ & \\
\hline 30-34 & 167 & -0.69 & $1 \cdot 36$ & 0.44 & $0.21,0.67$ & & $22 / 167$ & $13 \cdot 2$ & $0 \cdot 61$ & $0.36,1 \cdot 03$ & 0.53 & $0.29,0.96$ & \\
\hline$\geq 35$ & 76 & -0.67 & $1 \cdot 26$ & 0.46 & $0 \cdot 16,0.77$ & & $10 / 75$ & $13 \cdot 3$ & 0.62 & $0 \cdot 30,1 \cdot 27$ & 0.54 & $0 \cdot 24,1 \cdot 21$ & \\
\hline \multicolumn{14}{|l|}{ Mother's education } \\
\hline Secondary/tertiary & 695 & -0.64 & $1 \cdot 22$ & - & - & & $80 / 691$ & $11 \cdot 6$ & 1.00 & - & 1.00 & - & \\
\hline Primary/none & 784 & -0.98 & $1 \cdot 16$ & $-0 \cdot 28$ & $-0.41,-0.15$ & $<0.001$ & $129 / 782$ & $16 \cdot 5$ & $1 \cdot 51$ & $1 \cdot 12,2 \cdot 04$ & $1 \cdot 39$ & $1 \cdot 00,1 \cdot 93$ & 0.048 \\
\hline \multicolumn{14}{|l|}{ Mother's income (Ush) } \\
\hline$>60000$ & 102 & -0.36 & $1 \cdot 13$ & - & - & & $7 / 102$ & $6 \cdot 9$ & $1 \cdot 00$ & - & $1 \cdot 00$ & - & \\
\hline$\leq 60000$ & 1341 & -0.86 & $1 \cdot 19$ & -0.25 & $-0.50,-0.01$ & 0.058 & $196 / 1335$ & $14 \cdot 7$ & $2 \cdot 34$ & $1 \cdot 07,5 \cdot 11$ & $1 \cdot 79$ & $0.69,4.59$ & 0.197 \\
\hline \multicolumn{14}{|l|}{ Birth weight } \\
\hline Normal, $\geq 2.5 \mathrm{~kg}$ & 1382 & -0.77 & $1 \cdot 19$ & - & - & & $180 / 1377$ & $13 \cdot 1$ & $1 \cdot 00$ & - & 1.00 & - & \\
\hline Low, <2.5 kg & 89 & $-1 \cdot 55$ & $1 \cdot 19$ & $-0 \cdot 74$ & $-0.99,-0.48$ & $<0.001$ & $29 / 88$ & $33 \cdot 0$ & $3 \cdot 27$ & $2 \cdot 04,5 \cdot 24$ & $3 \cdot 14$ & $1 \cdot 86,5 \cdot 29$ & $<0.001$ \\
\hline \multicolumn{14}{|l|}{ Early weaning $\ddagger$} \\
\hline No & 1123 & $-0 \cdot 79$ & 1.22 & - & - & & $152 / 1117$ & $13 \cdot 6$ & 1.00 & - & 1.00 & - & \\
\hline Yes & 349 & -0.95 & $1 \cdot 13$ & $-0 \cdot 12$ & $-0.27,0.02$ & 0.099 & $58 / 349$ & $16 \cdot 6$ & $1 \cdot 27$ & $0.91,1 \cdot 76$ & $1 \cdot 20$ & $0 \cdot 84,1 \cdot 72$ & 0.314 \\
\hline \multicolumn{14}{|c|}{ Household socio-economic index§ } \\
\hline 1 (low) & 77 & -0.92 & 1.05 & - & - & 0.019 & $11 / 76$ & $14 \cdot 5$ & $1 \cdot 00$ & - & $1 \cdot 00$ & - & 0.408 \\
\hline 2 & 122 & $-1 \cdot 13$ & 1.06 & $-0 \cdot 23$ & $-0.57,0.11$ & 0.060 & $21 / 122$ & $17 \cdot 2$ & $1 \cdot 23$ & $0 \cdot 56,2 \cdot 72$ & 1.45 & $0.63,3.35$ & \\
\hline 3 & 451 & -0.92 & $1 \cdot 24$ & 0.02 & $-0.26,0.31$ & & $79 / 451$ & $17 \cdot 5$ & $1 \cdot 25$ & $0.63,2 \cdot 49$ & $1 \cdot 23$ & $0.59,2.56$ & \\
\hline 4 & 408 & -0.85 & 1.23 & -0.04 & $-0.33,0.25$ & & $60 / 407$ & $14 \cdot 7$ & 1.02 & $0.51,2 \cdot 05$ & $1 \cdot 26$ & $0 \cdot 60,2 \cdot 65$ & \\
\hline 5 & 312 & -0.52 & $1 \cdot 18$ & 0.20 & $-0.10,0.50$ & & $26 / 310$ & $8 \cdot 4$ & 0.54 & $0 \cdot 25,1 \cdot 15$ & $0 \cdot 80$ & $0 \cdot 36,1 \cdot 78$ & \\
\hline 6 (high) & 83 & -0.81 & $1 \cdot 02$ & -0.05 & $-0.43,0.33$ & & $8 / 81$ & $9 \cdot 9$ & 0.65 & $0 \cdot 25,1 \cdot 71$ & 0.99 & $0 \cdot 36,2 \cdot 76$ & \\
\hline \multicolumn{14}{|c|}{ Asymptomatic malaria at age 1 year } \\
\hline No & 1401 & $-0 \cdot 79$ & $1 \cdot 19$ & - & - & & 190/1397 & $13 \cdot 6$ & $1 \cdot 00$ & - & 1.00 & - & \\
\hline \multirow{2}{*}{\multicolumn{14}{|c|}{ Episodes of malaria during infancy }} \\
\hline & & & & & & & & & & & & & \\
\hline None & 1025 & -0.73 & $1 \cdot 19$ & - & - & $<0.001$ & $127 / 1021$ & $12 \cdot 4$ & $1 \cdot 00$ & - & $1 \cdot 00$ & - & 0.004 \\
\hline One & 256 & -0.95 & $1 \cdot 20$ & $-0 \cdot 18$ & $-0.34,-0.01$ & 0.001 & $41 / 255$ & $16 \cdot 1$ & $1 \cdot 35$ & $0.92,1.98$ & $1 \cdot 31$ & $0 \cdot 88,1.94$ & 0.001 \\
\hline Two or more & 172 & $-1 \cdot 23$ & $1 \cdot 20$ & -0.48 & $-0.67,-0.28$ & & $39 / 171$ & $22 \cdot 8$ & $2 \cdot 08$ & $1 \cdot 39,3 \cdot 11$ & $2 \cdot 12$ & $1 \cdot 38,3 \cdot 27$ & \\
\hline \multicolumn{14}{|c|}{ Maternal CD4 cell count } \\
\hline$>350$ cells $/ \mathrm{mm}^{3}$ & 60 & $-1 \cdot 04$ & $1 \cdot 37$ & - & - & & $12 / 60$ & $20 \cdot 0$ & $1 \cdot 00$ & - & $1 \cdot 00$ & - & \\
\hline$\leq 350$ cells $/ \mathrm{mm}^{3}$ & 21 & -0.93 & $1 \cdot 28$ & 0.00 & $-0.00,0.00$ & 0.261 & $4 / 21$ & $19 \cdot 1$ & 0.94 & $0 \cdot 27,3 \cdot 32$ & 4.09 & $0 \cdot 64,25 \cdot 9$ & 0.126 \\
\hline
\end{tabular}

LAZ, length-for-age Z-score; Ush, Uganda shillings.

Stunting: a chronic restriction of growth in length/height indicated by a low length-for-age $(L A Z<-2)$.

tAdjusted differences and odds ratios estimated from multivariate linear and logistic regression models that included maternal age, maternal education, income, household socio-economic status, episodes of malaria and

†Early weaning: introducing cow's milk at or before the age of 6 weeks

\$Household socio-economic status is a score based on building materials, number of rooms and items owned (' 1 ' representing lowest and ' 6 ' representing highest status). 
Table 3 Association of HIV exposure and other factors with underweight* in 1-year olds, Entebbe municipality and Katabi sub-county, Uganda, $2003-2005$

\begin{tabular}{|c|c|c|c|c|c|c|c|c|c|c|c|c|c|}
\hline \multirow[b]{2}{*}{ Risk factor } & \multirow[b]{2}{*}{$n$} & \multicolumn{2}{|c|}{ WAZ } & \multicolumn{2}{|c|}{ Difference in WAZ } & \multirow{2}{*}{$\begin{array}{c}P \text { value } \\
\text { (trend) }\end{array}$} & \multicolumn{2}{|c|}{ Underweight } & \multicolumn{4}{|c|}{ Odds for underweight } & \multirow{2}{*}{$\begin{array}{l}P \text { value } \\
\text { (trend) }\end{array}$} \\
\hline & & Mean & SD & Adjusted differencet & $95 \% \mathrm{Cl}$ & & $n / N$ & $\%$ & Unadjusted OR & $95 \% \mathrm{Cl}$ & Adjusted ORt & $95 \% \mathrm{Cl}$ & \\
\hline \multicolumn{14}{|l|}{ HIV exposure } \\
\hline HIV-unexposed & 1376 & -0.30 & $1 \cdot 16$ & - & - & & $102 / 1375$ & $7 \cdot 4$ & $1 \cdot 00$ & - & $1 \cdot 00$ & - & \\
\hline HIV-exposed & 122 & -0.45 & $1 \cdot 28$ & $-0 \cdot 13$ & $-0.35,0.09$ & 0.229 & $18 / 122$ & $14 \cdot 8$ & $2 \cdot 16$ & $1 \cdot 26,3 \cdot 70$ & $2 \cdot 32$ & $1.32,4.09$ & 0.006 \\
\hline \multicolumn{14}{|l|}{ Sex of child } \\
\hline Male & 763 & -0.41 & $1 \cdot 24$ & - & - & & $76 / 763$ & $10 \cdot 0$ & 1.00 & - & 1.00 & - & \\
\hline Female & 735 & -0.20 & $1 \cdot 13$ & $0 \cdot 22$ & $0 \cdot 10,0 \cdot 34$ & $<0.001$ & $44 / 734$ & $6 \cdot 0$ & 0.58 & $0 \cdot 39,0 \cdot 85$ & 0.57 & $0.38,0.85$ & 0.006 \\
\hline \multicolumn{14}{|c|}{ Mother's age group (years) } \\
\hline $14-19$ & 338 & -0.48 & $1 \cdot 13$ & - & - & 0.052 & $34 / 338$ & $10 \cdot 1$ & 1.00 & - & 1.00 & - & 0.609 \\
\hline $20-24$ & 562 & -0.32 & $1 \cdot 15$ & $0 \cdot 17$ & $0.01,0.33$ & 0.009 & $41 / 561$ & $7 \cdot 3$ & $0 \cdot 70$ & $0 \cdot 44,1 \cdot 13$ & $0 \cdot 70$ & $0 \cdot 42,1 \cdot 14$ & \\
\hline $25-29$ & 353 & -0.21 & $1 \cdot 13$ & $0 \cdot 22$ & $0.04,0.40$ & & $24 / 353$ & $6 \cdot 8$ & 0.65 & $0 \cdot 38,1 \cdot 13$ & $0 \cdot 67$ & $0 \cdot 37,1 \cdot 19$ & \\
\hline $30-34$ & 169 & -0.24 & $1 \cdot 23$ & $0 \cdot 18$ & $-0.04,0.41$ & & $15 / 169$ & 8.9 & 0.87 & $0.46,1.65$ & $0 \cdot 80$ & $0.39,1.62$ & \\
\hline$\geq 35$ & 76 & -0.07 & $1 \cdot 36$ & $0 \cdot 38$ & $0.08,0.67$ & & $6 / 76$ & $7 \cdot 9$ & 0.77 & $0 \cdot 31,1 \cdot 90$ & 0.67 & $0 \cdot 24,1 \cdot 82$ & \\
\hline \multicolumn{14}{|l|}{ Mother's education } \\
\hline Secondary/tertiary & 699 & $-0 \cdot 14$ & $1 \cdot 15$ & - & - & & $45 / 699$ & $6 \cdot 4$ & 1.00 & - & 1.00 & - & \\
\hline Primary/none & 795 & -0.46 & $1 \cdot 16$ & -0.21 & $-0.34,-0.09$ & 0.001 & $74 / 794$ & $9 \cdot 3$ & 1.49 & $1 \cdot 02,2 \cdot 20$ & $1 \cdot 33$ & $0.88,2 \cdot 02$ & $0 \cdot 173$ \\
\hline \multicolumn{14}{|l|}{ Mother's income (Ush) } \\
\hline$>60000$ & 101 & 0.33 & $1 \cdot 22$ & - & - & & $3 / 101$ & $3 \cdot 0$ & 1.00 & - & 1.00 & - & \\
\hline$\leq 60000$ & 1357 & -0.36 & $1 \cdot 14$ & -0.44 & $-0.69,-0.19$ & 0.001 & $113 / 1356$ & $8 \cdot 3$ & $2 \cdot 97$ & $0.93,9.52$ & 1.99 & $0.60,6.64$ & 0.222 \\
\hline \multicolumn{14}{|l|}{ Birth weight } \\
\hline Normal, $\geq 2.5 \mathrm{~kg}$ & 1397 & -0.26 & $1 \cdot 15$ & - & - & & $100 / 1396$ & $7 \cdot 2$ & 1.00 & - & 1.00 & - & \\
\hline Low, $<2.5 \mathrm{~kg}$ & 89 & -0.99 & $1 \cdot 16$ & -0.73 & $-0.98,-0.48$ & $<0.001$ & $18 / 89$ & $20 \cdot 2$ & $3 \cdot 29$ & $1 \cdot 88,5 \cdot 73$ & 3.00 & $1 \cdot 62,5 \cdot 53$ & 0.001 \\
\hline \multicolumn{14}{|l|}{ Early weaning } \\
\hline No & 1134 & -0.26 & $1 \cdot 15$ & - & - & & $77 / 1134$ & $6 \cdot 8$ & 1.00 & - & 1.00 & - & \\
\hline Yes & 353 & -0.47 & $1 \cdot 20$ & $-0 \cdot 17$ & $-0.31,-0.03$ & 0.020 & $43 / 352$ & $12 \cdot 2$ & 1.91 & $1 \cdot 29,2 \cdot 83$ & $1 \cdot 77$ & $1 \cdot 16,2 \cdot 71$ & 0.010 \\
\hline \multicolumn{14}{|c|}{ Household socio-economic index§ } \\
\hline 1 (low) & 80 & -0.37 & 1.02 & - & - & 0.011 & $3 / 80$ & $3 \cdot 8$ & 1.00 & - & 1.00 & - & $0 \cdot 128$ \\
\hline 2 & 122 & -0.58 & $1 \cdot 15$ & -0.20 & $-0.52,0.13$ & 0.012 & $12 / 122$ & $9 \cdot 8$ & $2 \cdot 80$ & $0 \cdot 76,10 \cdot 26$ & $2 \cdot 87$ & $0 \cdot 77,10 \cdot 74$ & \\
\hline 3 & 459 & -0.45 & $1 \cdot 20$ & -0.06 & $-0.34,0.21$ & & $48 / 458$ & $10 \cdot 5$ & $3 \cdot 00$ & $0.91,9.89$ & $3 \cdot 00$ & $0.90,9.99$ & \\
\hline 4 & 411 & -0.32 & $1 \cdot 15$ & -0.04 & $-0.32,0.24$ & & $33 / 411$ & $8 \cdot 0$ & $2 \cdot 24$ & $0.67,7 \cdot 49$ & $2 \cdot 50$ & $0.74,8.49$ & \\
\hline 5 & 312 & 0.00 & 1.07 & 0.21 & $-0.08,0.50$ & & $14 / 312$ & $4 \cdot 5$ & $1 \cdot 21$ & $0 \cdot 34,4 \cdot 30$ & 1.59 & $0 \cdot 44,5 \cdot 76$ & \\
\hline 6 (high) & 84 & -0.81 & 1.02 & 0.01 & $-0.36,0.37$ & & $8 / 84$ & $9 \cdot 5$ & $2 \cdot 70$ & $0.69,10.57$ & 3.69 & $0.92,14 \cdot 76$ & \\
\hline \multicolumn{14}{|c|}{ Asymptomatic malaria at age 1 year } \\
\hline No & 1416 & -0.29 & $1 \cdot 16$ & - & - & & $109 / 1415$ & $7 \cdot 7$ & $1 \cdot 00$ & - & $1 \cdot 00$ & - & \\
\hline Yes & 76 & -0.66 & $1 \cdot 19$ & $-0 \cdot 14$ & $-0.42,0.13$ & $0 \cdot 311$ & $10 / 76$ & $13 \cdot 2$ & $1 \cdot 82$ & $0.91,3.63$ & $1 \cdot 28$ & $0 \cdot 60,2 \cdot 73$ & 0.538 \\
\hline \multicolumn{14}{|c|}{ Episodes of malaria during pregnancy } \\
\hline None & 1034 & -0.24 & $1 \cdot 15$ & - & - & 0.008 & $75 / 1033$ & $7 \cdot 3$ & 1.00 & - & 1.00 & - & 0.0894 \\
\hline One & 258 & -0.40 & $1 \cdot 13$ & -0.13 & $-0.28,0.03$ & 0.002 & $20 / 258$ & $7 \cdot 8$ & 1.07 & $0.64,1.79$ & 1.03 & $0.61,1 \cdot 74$ & 0.052 \\
\hline Two or more & 176 & -0.58 & $1 \cdot 27$ & -0.28 & $-0.47,-0.09$ & & $24 / 176$ & $13 \cdot 6$ & $2 \cdot 02$ & $1 \cdot 23,3 \cdot 29$ & $1 \cdot 85$ & $1 \cdot 09,3 \cdot 14$ & \\
\hline \multicolumn{14}{|c|}{ Maternal CD4 cell count } \\
\hline$>350$ cells $/ \mathrm{mm}^{3}$ & 61 & -0.56 & $1 \cdot 27$ & - & - & & $9 / 61$ & $14 \cdot 8$ & 1.00 & - & $1 \cdot 00$ & - & \\
\hline$\leq 350$ cells $/ \mathrm{mm}^{3}$ & 21 & -0.41 & $1 \cdot 27$ & 0.00 & $-0.00,0.00$ & $0 \cdot 110$ & $5 / 21$ & $23 \cdot 8$ & $1 \cdot 81$ & $0.53,6 \cdot 17$ & $3 \cdot 13$ & $0 \cdot 61,16 \cdot 12$ & $0 \cdot 169$ \\
\hline
\end{tabular}

WAZ, weight-for-age Z-score; Ush, Uganda shillings.

*Underweight: a reflection of low weight-for-age (WAZ $<-2$ ).

tAdjusted differences and odds ratios estimated from multivariate linear and logistic regression models that included maternal age, maternal education, income, household socio-economic status, episodes of malaria and HIV exposure.

introducing cow's milk at or before the age of 6 weeks.

$\$$ Household socio-economic status is a score based on building materials, number of rooms and items owned (' 1 ' representing lowest and ' 6 ' representing highest status), 
Table 4 Association of HIV exposure and other factors with wasting* in 1-year olds, Entebbe municipality and Katabi sub-county, Uganda, 2003-2005

\begin{tabular}{|c|c|c|c|c|c|c|c|c|c|c|c|c|c|}
\hline \multirow[b]{2}{*}{ Risk factor } & \multirow[b]{2}{*}{$n$} & \multicolumn{2}{|c|}{ WLZ } & \multicolumn{2}{|c|}{ Difference in WLZ } & \multirow{2}{*}{$\begin{array}{c}P \text { value } \\
\text { (trend) }\end{array}$} & \multicolumn{2}{|c|}{ Wasting } & \multicolumn{4}{|c|}{ Odds for wasting } & \multirow{2}{*}{$\begin{array}{l}P \text { value } \\
\text { (trend) }\end{array}$} \\
\hline & & Mean & SD & Adjusted differencet & $95 \% \mathrm{Cl}$ & & $n / N$ & $\%$ & Unadjusted OR & $95 \% \mathrm{Cl}$ & Adjusted ORt & $95 \% \mathrm{Cl}$ & \\
\hline \multicolumn{14}{|l|}{ HIV exposure } \\
\hline HIV-unexposed & 1361 & $0 \cdot 13$ & $1 \cdot 17$ & - & - & & $52 / 1361$ & $3 \cdot 8$ & 1.00 & - & $1 \cdot 00$ & - & \\
\hline HIV-exposed & 121 & 0.03 & $1 \cdot 21$ & -0.04 & $-0 \cdot 27,0 \cdot 19$ & 0.741 & $5 / 121$ & $4 \cdot 1$ & 1.09 & $0 \cdot 43,2 \cdot 77$ & 0.97 & $0.37,2.52$ & 0.947 \\
\hline \multicolumn{14}{|c|}{ Number of living children } \\
\hline One & 409 & $0 \cdot 15$ & $1 \cdot 12$ & - & - & & $9 / 409$ & $2 \cdot 2$ & $1 \cdot 00$ & - & $1 \cdot 00$ & - & \\
\hline Two & 263 & -0.01 & $1 \cdot 17$ & -0.19 & $-0.39,0.01$ & $0 \cdot 132$ & $11 / 263$ & $4 \cdot 2$ & 1.94 & $0.79,4.75$ & $2 \cdot 38$ & $0 \cdot 92,6 \cdot 15$ & 0.019 \\
\hline Three & 167 & $0 \cdot 16$ & $1 \cdot 25$ & -0.04 & $-0.29,0.21$ & & $8 / 167$ & $4 \cdot 8$ & $2 \cdot 24$ & $0.85,5.90$ & $2 \cdot 34$ & $0.73,7.50$ & \\
\hline Four or more & 203 & 0.06 & $1 \cdot 33$ & -0.25 & $-0.53,0.03$ & & $17 / 203$ & $8 \cdot 4$ & $4 \cdot 06$ & $1 \cdot 78,9 \cdot 28$ & $6 \cdot 26$ & $1 \cdot 92,20 \cdot 45$ & \\
\hline \multicolumn{14}{|l|}{ Mother's income } \\
\hline$>60000$ & 101 & 0.66 & $1 \cdot 26$ & - & - & & $1 / 101$ & $1 \cdot 0$ & 1.00 & - & 1.00 & - & \\
\hline$\leq 60000$ & 1341 & 0.08 & $1 \cdot 16$ & -0.42 & $-0.68,-0.16$ & 0.002 & $55 / 1341$ & $4 \cdot 0$ & $4 \cdot 28$ & $0 \cdot 59,31 \cdot 23$ & $4 \cdot 41$ & $0.58,33.57$ & 0.074 \\
\hline \multicolumn{14}{|l|}{ Birth weight } \\
\hline Normal, $\geq 2.5 \mathrm{~kg}$ & 1382 & $0 \cdot 15$ & $1 \cdot 18$ & - & - & & $50 / 1382$ & $3 \cdot 6$ & $1 \cdot 00$ & - & $1 \cdot 00$ & - & \\
\hline Low, <2.5 kg & 88 & -0.32 & 1.09 & -0.52 & $-0.78,-0.26$ & $<0.001$ & $6 / 88$ & $6 \cdot 8$ & 1.95 & $0 \cdot 81,4 \cdot 68$ & $2 \cdot 31$ & $0.93,5 \cdot 71$ & 0.097 \\
\hline \multicolumn{14}{|l|}{ Early weaning $\ddagger$} \\
\hline No & 1122 & $0 \cdot 17$ & $1 \cdot 18$ & - & - & & $38 / 1122$ & $3 \cdot 4$ & 1.00 & - & 1.00 & - & \\
\hline Yes & 349 & -0.03 & $1 \cdot 18$ & $-0 \cdot 16$ & $-0.31,-0.01$ & 0.032 & $19 / 349$ & $5 \cdot 4$ & $1 \cdot 64$ & $0 \cdot 93,2 \cdot 89$ & $1 \cdot 35$ & $0 \cdot 73,2 \cdot 50$ & $0 \cdot 352$ \\
\hline \multicolumn{14}{|c|}{ Maternal CD4 cell count } \\
\hline$>350$ cells $/ \mathrm{mm}^{3}$ & 60 & -0.02 & $1 \cdot 15$ & - & - & & $2 / 88$ & $2 \cdot 3$ & & & & & \\
\hline$\leq 350$ cells $/ \mathrm{mm}^{3}$ & 21 & 0.06 & $1 \cdot 31$ & 0.00 & $-0.00,0.00$ & $0 \cdot 154$ & $0 / 21$ & 0 & & & & & \\
\hline
\end{tabular}

WLZ, weight-for-length Z-score.

Wasting: an acute weight loss indicated by a low weight-for-length (WLZ $<-2)$.

tAdjusted differences and odds ratios estimated from multivariate linear and logistic regression models that included maternal age, maternal education, income, household socio-economic status, episodes of malaria and HIV exposure.

¥Early weaning: introducing cow's milk at or before the age of 6 weeks. 
that $28 \%$ of HIV-positive mothers had a CD 4 count of $\leq 350$ cells $/ \mathrm{mm}^{3}$, representing a category of mothers who qualify for antiretroviral therapy (ART) according to the most recent recommendations ${ }^{(23)}$; low maternal CD 4 cell count increased the odds of both stunting and being underweight (although weakly), but was not associated with wasting.

It has been suggested that infections may be more common among HIV-exposed uninfected children; if so, such infections could contribute to poor growth ${ }^{(24)}$, but this was not the case in our study. The study clinic provided cotrimoxazole prophylaxis to all HIV-exposed uninfected infants and this could have helped to diminish infection risks. In multivariable models, following our modelling strategy we chose to adjust for malaria episodes in infancy as it was strongly associated with growth outcomes and hence may have improved the precision of estimates from linear regression. It is also likely to be a proxy for maternal malaria (which we considered likely to be a confounder of the association between HIV exposure and growth outcomes). Theoretically it is possible that malaria in infancy could be on the causal pathway between HIV exposure and growth outcomes, but this was not the case in our study due to the provision of cotrimoxazole for all HIV-exposed infants, so that HIV exposure was not in fact associated with infant malaria. Therefore inclusion of malaria episodes in infancy in the multivariable model had little impact on the adjusted associations between HIV exposure and growth outcomes.

Contrary to some of our study findings, researchers in Europe $^{(2)}$ reported that exposure to maternal HIV infection is not associated with child's poor growth. This difference could be due to the fact that in Europe the HIVinfected population is more like the general population and less socio-economically disadvantaged than that in Uganda. This suggests that the associations that we and researchers in Zambia ${ }^{(5)}$ have observed in HIV-exposed but uninfected children may not be due to the infection itself, but rather to concomitant social or behavioural factors. It is also possible that the association we have observed between HIV exposure and underweight is a chance finding; this possibility is supported by the fact that analysis of the corresponding continuous WHZ outcome did not show an association with HIV exposure.

Uninfected children born to HIV-infected women are exposed antenatally to ART and this might affect their growth in early life. A study conducted in Thailand found that, although neither the total duration of exposure to zidovudine nor the duration of postnatal exposure was associated with infant growth from 6 weeks to 18 months, a longer in utero exposure to zidovudine had a negative impact on birth weight ${ }^{(25)}$. A study in Europe found a minimal effect of combination ART on growth up to 18 months of age ${ }^{(26)}$. Other findings in Europe showed that ART exposure was not significantly associated with prevalence of congenital abnormalities or low birth weight ${ }^{(27)}$.
But ART exposure was associated with anaemia in infants, with $\mathrm{Hb}$ levels reverting to normal by 3 months of age ${ }^{(27)}$. However, in our setting, single-dose nevirapine for mother and child was standard of care at the time of the study and prolonged exposure of the HIV-exposed but uninfected infants to ART in infancy or in utero was rare.

Strengths of the study are its large sample size and cohort design, meaning that data on exposures of interest and potential confounders were collected prospectively. Possible limitations of our study include the fact that the mothers and infants included in the present analysis were not representative of the study population, with children of HIV-infected women being less likely to provide growth data than children of HIV-uninfected women. Moreover, since HAART regimens were not standard of care during pregnancy in the study setting at the time, we are unable to comment on the impact of triple HAART regimens on infant growth.

On the other hand, in our study (as recommended in this setting), HIV-exposed but uninfected infants routinely received cotrimoxazole from 6 weeks to 18 months of age: a folate antagonist which can cause anaemia and might possibly interfere directly with growth. We found that HIV-exposed uninfected babies were more anaemic than HIV-unexposed, but the difference was not significant (data not shown).

As regards determinants of stunting or underweight other than HIV exposure, our results complement findings of previous studies ${ }^{(13,28)}$ that being a male child is associated with a higher risk of both stunting and underweight. Other studies have also found that young maternal age is a strong predictor for stunting and being underweight $^{(29)}$ although results are not consistent ${ }^{(13)}$. Low maternal education, low birth weight and early weaning were associated with increased odds of stunting and underweight in our study, consistent with findings of earlier studies ${ }^{(9,30,31)}$. As expected, the odds of stunting and being underweight increased significantly with the number of malaria episodes in infancy. This is in agreement with earlier reports that episodes of illness are associated with appetite loss, vomiting and poor nutrient utilization, which exacerbates malnutrition and perpetuates the cycle $^{(32)}$. Number of living children was not associated with stunting or underweight but significantly increased the odds of wasting, consistent with the hypothesis that the oldest child is likely to be the least malnourished and that subsequent children are increasingly poorly provided for.

\section{Conclusions}

Maternal HIV infection was associated with being underweight in HIV-uninfected infants in this population. The underlying mechanism is not fully understood and may be multifactorial. Additional independent predictors of stunting or underweight in this population included 
young maternal age, low maternal education, low birth weight and malaria in infancy. In addition to effective interventions for prevention of mother-to-child HIV transmission, children born to HIV-infected mothers also require special care to ensure that their growth and development are not impaired. Prevention and treatment of undernutrition in children remain critical interventions in settings with high HIV prevalence.

\section{Acknowledgements}

Sources of funding: The study was funded by the Wellcome Trust (grant number 064693). Conflicts of interest: The authors have no conflict of interest to declare. Ethics: Ethical approval for the Entebbe trial of de-worming during pregnancy was given by the Science and Ethics Committee, Uganda Virus Research Institute; the Uganda National Council for Science and Technology; and the London School of Hygiene \& Tropical Medicine. Authors' contributions: S.A.L., H.M., J.N. and M.N. carried out the interviews, collected the data and contributed to revising the paper. M. Kizza and E.N. facilitated data collection and supported the fieldwork. M. Kihembo supported laboratory work especially the measurement of maternal CD4 cell counts. L.M. planned and undertook the analysis, undertook the interpretation of the data and drafted the manuscript. E.L.W. contributed to analysis, interpretation of data and revising the paper. A.M.E. designed the study, provided overall research supervision, and contributed to analysis, interpretation of data and revising the paper. Acknowledgements: The authors thank the study participants, the fieldwork team, Entebbe Hospital and the MRC/UVRI Uganda Research Unit on AIDS for their support. They also thank Professor Jonathan Levin who gave useful technical comments and advice.

\section{References}

1. Webb AL, Manji K, Fawzi WW et al. (2009) Timeindependent maternal and infant factors and timedependent infant morbidities including HIV infection, contribute to infant growth faltering during the first 2 years of life. J Trop Pediatr 55, 83-90.

2. Newell ML, Borja MC \& Peckham C (2003) Height, weight and growth in children born to mothers with HIV-1 infection in Europe. Pediatrics 111, e52-e60.

3. Arpadi S, Fawzy A, Aldrovandi GM et al. (2009) Growth faltering due to breastfeeding cessation in uninfected children born to HIV-infected mothers in Zambia. Am J Clin Nutr 90, 344-353.

4. Gangar J (2009) Nutritional assessment of newborns of HIV-infected mothers. Indian Pediatr 46, 339-341.

5. Makasa M, Kasonka L, Chisenga M et al. (2007) Early growth of infants of HIV-infected and uninfected Zambian women. Trop Med Int Health 12, 594-602.

6. Agostoni C, Zuccotti GV, Giovannini M et al. (1998) Growth in the first two years of uninfected children born to HIV-1 seropositive mothers. Arch Dis Child 79, 175-178.
7. Kuhn L, Kasonde P, Sinkala M et al. (2005) Does severity of HIV disease in HIV-infected mothers affect mortality and morbidity among their uninfected infants? Clin Infect Dis 41, 1654-1661.

8. Ezeaka VC, Iroha EO, Akinsulie AO et al. (2009) Anthropometric indices of infants born to HIV-1-infected mothers: a prospective cohort study in Lagos, Nigeria. Int J STD AIDS 20, 545-548.

9. Cattaneo A, Timmer A, Bomestar T et al. (2008) Child nutrition in countries of the Commonwealth of Independent States: time to redirect strategies? Public Health Nutr 11, 1209-1219.

10. de Villiers A \& Senekal M (2002) Determinants of growth failure in 12-24-month-old children in a high-density urban slum community in East London, South Africa. Eur J Clin Nutr 56, 1231-1241.

11. Hong R \& Mishra V (2006) Effect of wealth inequality on chronic under-nutrition in Cambodian children. $J$ Health Popul Nutr 24, 89-99.

12. Kikafunda JK, Walker AF, Collett D et al. (1998) Risk factors for early childhood malnutrition in Uganda. Pediatrics 102, E45.

13. Hien NN \& Hoa NN (2009) Nutritional status and determinants of malnutrition in children under three years of age in Nghean, Vietnam. Pakistan J Nutr 8, 958-964.

14. Patel V, Rahman A, Jacob KS et al. (2004) Effect of maternal mental health on infant growth in low income countries: new evidence from South Asia. BMJ 328, 820-823.

15. Kikafunda KK, Namusoke HK (2006) Nutritional status of HIV/AIDS orphaned children in households headed by the elderly in Rakai District, South Western, Uganda. Afr J Food Agric Nutr Dev 6, issue 1; available at http://www.ajol.info/ index.php/ajfand/article/download/19169/62500

16. Elliott AM, Kizza M, Quigley MA et al. (2007) The impact of helminths on the response to immunization and on the incidence of infection and disease in childhood in Uganda: design of a randomized, double-blind, placebo-controlled, factorial trial of deworming interventions delivered in pregnancy and early childhood [ISRCTN32849447]. Clin Trials 4, 42-57.

17. Ndibazza J, Muhangi L, Akishule D et al. (2010) Effects of deworming during pregnancy on maternal and perinatal outcomes in Entebbe, Uganda: a randomized controlled trial. Clin Infect Dis 15, 531-540.

18. Webb EL, Mawa PA, Ndibazza J et al. (2011) Effect of singledose anthelmintic treatment during pregnancy on an infant's response to immunisation and on susceptibility to infectious diseases in infancy: a randomised, double-blind, placebo-controlled trial. Lancet 377, 52-62.

19. Guay LA, Musoke P, Fleming $T$ et al. (1999) Intrapartum and neonatal single-dose nevirapine compared with zidovudine for prevention of mother-to-child transmission of HIV-1 in Kampala, Uganda: HIVNET 012 randomised trial. Lancet 354, 795-802.

20. Muhangi L, Woodburn P, Omara M et al. (2007) Associations between mild-to-moderate anaemia in pregnancy and helminth, malaria and HIV infection in Entebbe, Uganda. Trans R Soc Trop Med Hyg 101, 899-907.

21. Kapetanovic S, Christensen S, Karim R et al. (2009) Correlates of perinatal depression in HIV-infected women. AIDS Patient Care STDS 23, 101-108.

22. Stewart RC (2007) Maternal depression and infant growth: a review of recent evidence. Matern Child Nutr 3, 94-107.

23. World Health Organization (2010) Antiretroviral therapy for HIV infection in adults and adolescents: Recommendations for a public health approach, 2010 revision. http:// whqlibdoc.who.int/publications/2010/9789241599764_eng.pdf (accessed May 2012).

24. Filteau S (2009) The HIV-exposed, uninfected African child. Trop Med Int Health 14, 276-287. 
25. Briand N, Le Coeur S, Traisathit P et al. (2006) Growth of human immunodeficiency virus-uninfected children exposed to perinatal zidovudine for the prevention of mother-to-child human immunodeficiency virus transmission. Pediatr Infect Dis J 25, 325-332.

26. Hankin C, Thorne C, Newell ML et al. (2005) Does exposure to antiretroviral therapy affect growth in the first 18 months of life in uninfected children born to HIVinfected women? Acquir Immune Defic Syndr 40, 364-370.

27. European Collaborative Study (2003) Exposure to antiretroviral therapy in utero or early life: the health of uninfected children born to HIV-infected women. J Acquir Immune Defic Syndr 32, 380-387.

28. Kabubo-Mariara J, Ndenge GK \& Mwabu DK (2008) Determinants of children's nutritional status in Kenya: evidence from demographic and health surveys. J Afr Econ 18, 363-387.
29. Linnemayr S, Alderman H \& Ka A (2008) Determinants of malnutrition in Senegal: individual, household, community variables, and their interaction. Econ Hum Biol 6, 252-263.

30. Bloss E, Wainaina F \& Bailey RC (2004) Prevalence and predictors of underweight, stunting, and wasting among children aged 5 and under in western Kenya. J Trop Pediatr 50, 260-270.

31. Mamiro PS, Kolsteren P, Roberfroid D et al. (2005) Feeding practices and factors contributing to wasting, stunting, and iron-deficiency anaemia among 3-23-month old children in Kilosa district, rural Tanzania. J Health Popul Nutr 23, 222-230.

32. Devereux S, Baulch B, Phiri A et al. (2006) Vulnerability to Chronic Poverty and Malnutrition in Malawi. A report for DFID Malawi. http://www.ids.ac.uk/files/MalawiVulnerability Report_Final.pdf (accesed May 2012). 